\title{
A new simplified fluid dynamic model for digital twins of electrohydraulic servovalves
}

\author{
Matteo Davide Lorenzo Dalla Vedova and Pier Carlo Berri \\ Department of Mechanical and Aerospace Engineering, Politecnico di Torino, Turin, Italy
}

\begin{abstract}
Purpose - The purpose of this paper is to propose a new simplified numerical model, based on a very compact semi-empirical formulation, able to simulate the fluid dynamics behaviors of an electrohydraulic servovalve taking into account several effects due to valve geometry (e.g. flow leakage between spool and sleeve) and operating conditions (e.g. variable supply pressure or water hammer).

Design/methodology/approach - The proposed model simulates the valve performance through a simplified representation, deriving from the linearized approach based on pressure and flow gains, but able to evaluate the mutual interaction between boundary conditions, pressure saturation and leak assessment. Its performance was evaluated comparing with other fluid dynamics numerical models (a detailed physics-based high-fidelity one and other simplified models available in the literature).

Findings - Although still showing some limitations attributable to its simplified formulation, the proposed model overcomes several deficiencies typical of the most common fluid dynamic models available in the literature, describing the water hammer and the nonlinear dependence of the delivery differential pressure with the spool displacement.

Originality/value - Although still based on a simplified formulation with reduced computational costs, the proposed model introduces a new nonlinear approach that, approximating with suitable precision the pressure-flow fluid dynamic characteristic of a servovalve, overcomes the shortcomings typical of such models.

Keywords Monitoring, Simulation, Digital twin, Aerospace systems, EHA, Flight commands, Lumped parameters, Nonlinear modeling, Servovalve, Simplified fluid dynamic numerical models
\end{abstract}

Paper type Research paper

\section{List of symbols}

$C_{L k} \quad=$ leakage coefficient $\left(\mathrm{m}^{3} / \mathrm{s} / \mathrm{Pa}\right)$;

Com = servomechanism position command (m);

$d x_{\mathcal{f}} \quad=$ motor element velocity $(\mathrm{m} / \mathrm{s})$;

EHA = electrohydraulic actuator;

$\mathrm{EHSV}=$ electrohydraulic servovalve;

Err = position error $\left(E r r=\operatorname{Com}-x_{\mathcal{F}}\right)(\mathrm{m})$;

$F_{R} \quad=$ load acting on the motor element $(\mathrm{N})$;

$G_{P} \quad=$ pressure gain $(\mathrm{Pa} / \mathrm{m})$;

$G_{Q} \quad=$ flow gain $\left(\mathrm{m}^{2} / \mathrm{s}\right) ;$

$G_{P Q}=$ pressure to flow gain ratio $G_{P} / G_{Q}\left(\mathrm{~Pa} \cdot \mathrm{s} / \mathrm{m}^{3}\right) ;$

$i_{c} \quad=$ reference current signal of the valve (A);

$P_{12}=$ actual differential pressure $(\mathrm{Pa}) ;$

$P_{12 P}=$ zero-flow controlled differential pressure $(\mathrm{Pa})$;

$P_{S R} \quad=$ supply/return differential pressure $(\mathrm{Pa})$;

$P_{\text {vap }}=$ hydraulic fluid vapor tension value $(\mathrm{Pa})$;

$Q_{L k} \quad=$ leakage flow $\left(\mathrm{m}^{3} / \mathrm{s}\right) ;$

$Q_{f} \quad=$ working flow $\left(\mathrm{m}^{3} / \mathrm{s}\right)$;

$x_{\mathcal{F}} \quad=$ motor element position $(\mathrm{m})$;

$x_{S} \quad=$ spool displacement $(\mathrm{m})$;

$x_{S M}=$ spool end of travel displacement $(\mathrm{m})$;

$x_{S S}=P_{12 P}$ saturation spool displacement $(\mathrm{m})$; and

$x_{S t} \quad=$ equivalent spool position $(\operatorname{model} \mathrm{C} 5)(\mathrm{m})$.

The current issue and full text archive of this journal is available on Emerald Insight at: https://www.emerald.com/insight/1748-8842.htm

Aircraft Engineering and Aerospace Technology 94/1 (2022) 79-88

Emerald Publishing Limited [ISSN 1748-8842] [DOI 10.1108/AEAT-12-2020-0321]

\section{Introduction}

Although in recent decades, alternative actuation systems, frequently based on electric machines, are progressively establishing themselves, hydraulic actuators still play a crucial role in several technological sectors (e.g. fluid automation, mechatronics, servomechanisms and aerospace) and, in particular, for flight controls (as reported by De Martin et al., 2018b). Nowadays, electrohydraulic (EH) servomechanisms are still widely used within current generation aircraft flight control systems, as they provide power density unmatched by alternative technologies and very high reliability (De Martin et al., 2018a). Among the most common applications in aerospace technology, $\mathrm{EH}$ actuators are used for powering flyby-wire aerodynamic surfaces, landing gear retraction, steering and braking as well as several secondary users. However, to match the safety requirements for the use in commercial and military aviation, redundancies and health monitoring strategies are usually needed (Byington et al., 2004). The development of electrohydraulic actuators (EHA) for the

(C) Matteo Davide Lorenzo Dalla Vedova and Pier Carlo Berri. Published by Emerald Publishing Limited. This article is published under the Creative Commons Attribution (CC BY 4.0) licence. Anyone may reproduce, distribute, translate and create derivative works of this article (for both commercial \& non-commercial purposes), subject to full attribution to the original publication and authors. The full terms of this licence may be seen at http://creativecommons.org/licences/by/4.0/legalcode

Received 31 December 2020

Revised 9 March 2021

Accepted 27 April 2021 
control of aerodynamic aircraft surfaces often requires the use of accurate and high-fidelity fluid dynamic numerical simulations that able to evaluate the performance of the system within its operating envelope (Borello et al., 2009). In this regard, several works are available in the literature (Chen and Stoffel, 2004; Mehring et al., 2014). For instance, Urata investigated the effect of various failure modes on the behavior of hydraulic servovalves, namely, leakage flow, fringing, eddy currents and air gap asymmetry in the torque motor (Urata, 2000, 2001, 2002, 2003, 2004a, 2004b, 2005). Other detailed analyses of the behavior of servovalves are proposed by Yang et al. (2019) and Henninger et al. (2017) using computational fluid dynamics (CFD) and electromagnetic finite elements, respectively.

However, these detailed simulations are generally costly, both in terms of computational time and processor workload and therefore can be unsuitable for certain tasks, whose execution is time or resource constrained. In this category lies, for example, the preliminary design of the systems as well as the development and implementation of real-time systems (e.g. monitoring and diagnostic routines). The challenge in these cases lies in achieving a suitable level of accuracy while keeping the computational burden low at the same time. Therefore, it is necessary to create simplified models, conceived and adequately set for specific operations such as the optimization of the preliminary design and the development of diagnostic or prognostic strategies to use as digital twins of the real system where necessary (as proposed by Dalla Vedova et al., 2019).

These simplified numerical models must often combine sufficient levels of accuracy and reliability with reduced computational costs and real-time performance. EHAs have been in use for many decades but are still critical in aerospace systems today. Their sensitivity to various failure modes, the difficulty of promptly identifying these malfunctions and the possible consequences (in terms of safety for the entire system) oblige to prepare the appropriate tools to model and monitor the system and its various components. In particular, it is needed to conceive new dedicated numerical models of the electrohydraulic servovalves (EHSVs) that control these systems, capable of combining an acceptable computational effort with a satisfying ability to simulate their performance and the most peculiar dynamic behaviors. In this regard, this article presents a new simplified numerical model, based on a very compact semi-empirical formulation, simulating the fluid dynamics of the servovalve. Still, it can evaluate, in a simplified but adequately accurate way, several typical behaviors related to the geometry of the valve drawer (e.g. flow leakage between spool and sleeve) and the operating conditions (e.g. variable supply pressure or water hammer). The behaviors of the proposed model are assessed by comparing their results against a detailed physics-based highfidelity (HF) model proposed by Borello et al. (2009). This HF model computes the response of the equipment accounting for the pressure-flow characteristics across all the internal passageways of the valve second stage (i.e. the regulating drawer composed by spool and sleeve).

\section{Electrohydraulic actuator general architecture}

In the aerospace field, and especially in aircraft onboard applications, electrohydraulic actuators are still very widely used. Therefore, as reported in the technical literature by Maré $(2016,2018)$, it is possible to find many different architectures and related variants (related, for example, to the fields of application, performance and interface requirements and redundancy levels).

The simplest and most basic architecture of an EHA, according to Dalla Vedova et al. (2014), includes the following main three elements. An electronic control unit compares the instantaneous error between the current position of the actuator and the corresponding setpoint and calculates the current input feeding the control valve. The said input current flows through the torque motor moving the first stage flapper toward one of the nozzles. This displacement unbalances the pilot pressures acting on either side of the second stage of the EHSV and causes the spool to move from its null position (opening or closing the fluid passages on the sleeve). Eventually, the spool displacement, through the feedback spring, draws the flapper back to an equilibrium position, equalizing the pressures on the spool (according to Jacazio and Borello, 1986).

The EHSV (usually of flapper-nozzle type with a two-stage and four-way design) is the regulating element that routes the hydraulic fluid to the control ports of the motor element, typically a linear jack or a rotary hydraulic motor, which converts hydraulic power into mechanical power to move the user. (e.g. a control surface of the aircraft). It should be noted that EHSV is generally the most complex (and safety-critical) element of EHA and, therefore, appropriate modeling of its performance is crucial for the study of such systems. The design for a typical aerospace EHA, with related two-stage, four-way, flapper-nozzle EHSV, is shown in Figure 1(a).

\section{Simulated electrohydraulic actuator test bench}

A simulated test bench was developed to compare the performances of different servovalve models by integrating them into a numerical simulation of the operation of a typical electrohydraulic actuator for flight controls. The numerical test bench reflects the servoactuator architecture depicted in Figure 1. A torque motor drives the first-stage flapper-nozzle valve by moving the flapper closer to one of the two nozzles. As a result, the pressure loss across the two nozzles becomes unbalanced and causes a differential pressure to arise on the sides of the second stage spool. The spool moves under the effect of this differential pressure until it brings the flapper back in the center position.

The spool position $x_{S}$ rules the opening of the second stage control passageways 1 and 2, connecting one of the corresponding ports to the supply pressure $S$ and the other to the return pressure R. The differential pressure $P_{12}$ varies progressively from $P_{S R}=$ $P_{S}-P_{R}$ to $-P_{S R}$, depending on the spool position and the flow rate. This differential pressure, multiplied by the active area of the hydraulic jack, gives the motor force applied to the piston. The motion of the user assembly as a result of this hydraulic force is computed by a nonlinear, second-order dynamical system accounting for dry friction, endstops and backlash.

\subsection{Detailed electrohydraulic servovalves numerical model}

A HF model relying on a one-dimensional computational fluid dynamics (CFD) simulation serves as a baseline for evaluating the performances of the considered simplified servovalve models 
Figure 1 (a) Schematic of an EHA equipped with a two-stage, four-way, flapper-nozzle EHSV; and (b) pressure-flow rate-spool position characteristic of the SV, obtained through the HF model

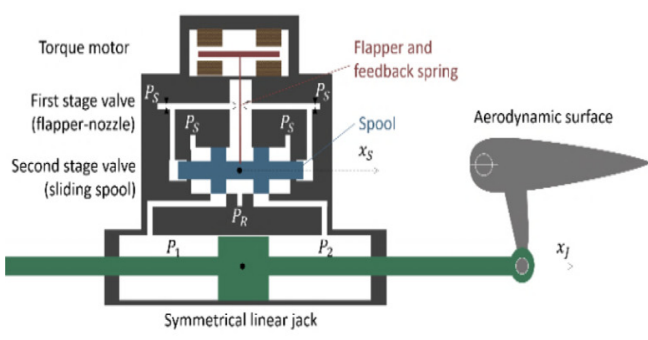

(a)

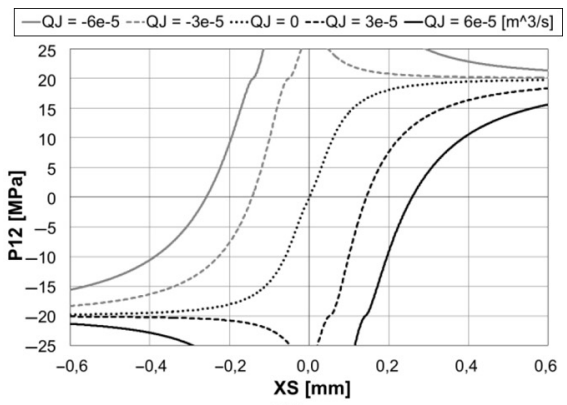

(b)
(Borello et al., 2009). Its accuracy has been validated by comparison with experimental data and CFD simulations (Dalla Vedova, 2007; Pace et al., 2013), and its behavior is shown in the fluid dynamics map of Figure 2. The model, developed in Fortran and Simulink by Berri and Dalla Vedova (2020), computes the pressure drops because of the fluid crossing the restriction caused by the four passageways of the valve. The actual geometry of the spool and sleeve is accounted for, including the spool-sleeve radial clearance, the valve over/underlapping, and the shape of the passageways. For zero flow rate and small spool displacements $x_{S}$, the pressure-flow curve follows a linear relationship. As $x_{S}$ approaches its saturation value $x_{S S}$, the differential pressure settles to the supply value $\pm P_{S R}$. The presence of a nonzero flow rate shifts this curve approximately linearly. Additionally, if a flow rate $Q_{\mathcal{f}} \neq 0$ is forced to cross a closed valve (i.e. for $x_{S} \approx 0$ ) the pressure rises steeply, even higher than the supply differential pressure. This effect, which increases proportionally to the oil flow imposed through the valve regulation ports, is commonly known as "water hammer." It often plays a meaningful role in the actuator response (e.g. system behaviors, dynamic damping and related stiffness, structural damages because of the fatigue on the valve and hydraulic circuit). The HF model is quite computationally intensive and highly dependent on a large number of parameters, for example, related to the physical properties of the working fluid and the geometrical tolerances of the valve components. Some of these parameters are subject to variations during the operation of the system (e.g. the temperature of the fluid may change, or the spool-sleeve clearances may vary as a result of pressure-induced elastic deformation); then, lower fidelity models are often required to simplify system-level simulations.

\section{Simplified electrohydraulic servovalves numerical models}

The simplest servovalve models available in literature leverage a two-gains linear formulation. On the base of this simplified approach, the valve is modeled on the base of the simple relations between input and output signals. The relationship between flow rate $Q_{\mathcal{f}}$, differential pressure $P_{12}$ and spool displacement $x_{S}$ is expressed as a function of a flow gain $G_{Q}$ and a pressure gain $G_{P}$, both of which can be measured experimentally considering the valve as a blackbox:

$$
P_{12}=G_{P}\left(x_{S}-Q_{f} / G_{Q}\right)
$$

Clearly, this formulation is applicable only for small spool displacements and for low values of the differential pressure (i.e. near the origin of the fluid dynamic map of Figure 2): the pressure saturation due to the limited supply is not accounted for, and the operation of the actuator cannot be simulated near its stall load condition. Additionally, the water hammer effect, transitions between laminar and turbulent conditions, and leakage through the valve clearances are neglected.

Figure 2 Block diagrams of the simplified EHSV models from the literature

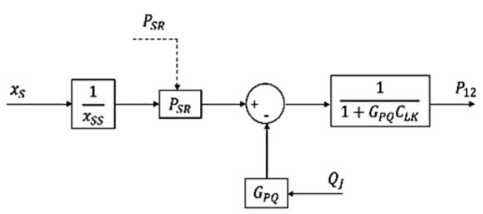

(a)

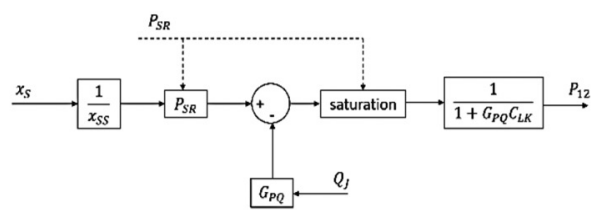

(b)

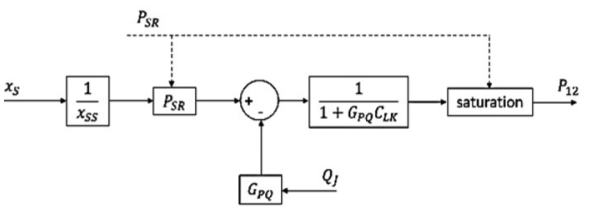

(c)

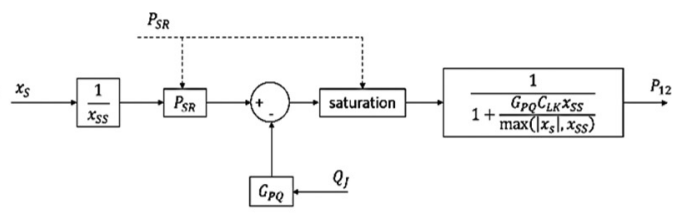

(d) 
The shortcomings because of the linearized models usually make them inadequate for real-time health monitoring duties, as the differences between the numerical model and physical system can trigger false positive fault detections. In this context, several iterations of low fidelity models were developed to include the effects of pressure saturation, leakage flows and water hammer effects into the simulation. The following paragraph describes these models.

\subsection{Model a}

Model A was first proposed by Borello and Villero (1991). It can take into account a variable supply differential pressure $P_{S R}$, as well as for the leakage across the clearance between the spool and sleeve of the valve, through the leakage coefficient $C_{L K}$. The pressure gain $G_{P}$ can usually be considered proportional to the supply pressure differential. As a matter of fact, their ratio $x_{S S}=P_{S R} / G_{P}$ can be assumed approximately constant with respect to supply pressure and is physically equivalent to the spool displacement needed to saturate the pressure output of the valve in zero flow conditions. The flow gain $G_{Q}$ is proportional to the square root of $P_{S R}$ if assuming a fully turbulent formulation. However, given the linear formulation of the proposed simplified models, a linear approximation can be assumed between $G_{Q}$ and $P_{S R}$ (as introduced by Alimhillaj et al., 2013).

As a result, the quantity $G_{P Q}=G_{P} / G_{Q}$ can be assumed as nearly constant. Additionally, an internal leakage flow $Q_{L K}=$ $C_{L K} P_{12}$ recirculating between the two control ports 1 and 2 can be considered as proportional to the differential pressure $P_{12}$. This computation creates an instantaneous loop, as the leakage flow depends on the differential pressure, and the differential pressure, in turn, depends on the total flow $Q_{f}+Q_{L K}$. To avoid numerical problems, the loop can be pre-solved, resulting in the transfer function:

$$
P_{12}=\left(\frac{P_{S R}}{x_{S S}} x_{S}-Q_{f} G_{P Q}\right) \frac{1}{1+G_{P Q} C_{L K}}
$$

Model A is summarized by the block diagram of Figure 2(a). Although the sensitivity to supply pressure variations and to leakage flows has been introduced, the model remains completely linear and unable to account for pressure saturations and water hammer effects. Then, the models C1, C2 and C3 are progressive iterations intended to add such sensitivity to the simulation.

\subsection{Model C1}

As proposed by Alimhillaj et al. (2013), the model C1 derives from a modification of model A aiming to add sensitivity to the pressure saturation. Indeed, neglecting the water hammer effect, a valve can normally provide a maximum differential pressure close to (or, at most, equal to) the supply differential pressure provided by the hydraulic circuit. Model C1, with a layout similar to model $\mathrm{A}$, has a saturation block upstream the leakage evaluation, as shown in the block diagram of Figure 2(b). The pre-solved leakage loop is evaluated after the saturation. This way, the maximum pressure differential produced by the valve is limited to $P_{S R}$, and the leakage flow is assimilated to the output flow $Q_{f}$. As $G_{P Q} C_{L K}>0$, the gain of the leakage loop is less than unit, and the architecture of the model bounds the output pressure to be less than the supply differential pressure: specifically, $\left|P_{12}\right| \leq P_{S R} /\left(1+G_{P Q} C_{L K}\right)$.

\subsection{Mode1 C2}

The EHSV numerical model C2, also proposed by Alimhillaj et al. (2013), is represented by the block diagram shown in Figure 2(c). It is obtained from model $\mathrm{C} 1$ by switching places between the leakage flow and saturation block. This way, model $\mathrm{C} 2$ addresses one of the limits of model $\mathrm{C} 1$; that is, it allows the control differential pressure $P_{12}$ to rise up to the supply differential pressure $P_{S R}$.

As a result, this formulation permits to predict within acceptable accuracy the behavior of the actuator near stall conditions, as well as the limitation in the maximum no-load actuation speed because of the finite supply pressure. Although, the model may fail to correctly represent the effect of temporary overloads of the servosystem, as it neglects the (sometimes significant) pressure rise because of the water hammer effect. In particular, both models C1 and C2 will overestimate the speed at which the actuator recesses under the effect of an external force higher than its stall load. As these conditions are not usually encountered during the normal operation of electrohydraulic flight control systems, this limitation may still be acceptable for some applications.

\subsection{Model C3}

The EHSV numerical model C3, already introduced by Alimhillaj and Dalla Vedova (2018), is also derived from the model $\mathrm{C} 1$ and, compared to the previous ones, tries to model with greater accuracy the interaction between the different nonlinearities that characterize the system. Similarly to model C1, model C3 keeps the pressure saturation upstream the presolved leakage loop. However, the latter features a modified formulation to account for the pressure saturation within the leakage flow evaluation, by means of the saturation value $x_{S S}$ of the spool displacement $x_{S}$.

Specifically, the $G_{P Q} C_{L K}$ contribution of the leakage flow is reduced through a coefficient $x_{S S} /\left|x_{S}\right|$ only when $\left|x_{S}\right|>x_{S S}$, that is, when the spool displacement is large. This way, for large spool displacements the output control pressure $P_{12}$ can reach the supply pressure differential $P_{S R}$, regardless the leakage flow. The block diagram of model C3 is shown in Figure 2(d).

\subsection{Other numerical models simulating the fluid dynamic behavior of the valve}

Despite the subsequent improvements, the models presented in the previous sections are not, however, able to describe with sufficient accuracy the peculiar nonlinear phenomena characterizing the fluid dynamics of the valve. Therefore, in some previous works, the authors have already faced these topics and proposed alternative solutions, aiming to overcome the limits mentioned above and arrive at a formulation capable of reconciling a satisfactory level of accuracy with reduced computational costs. The first evolution of the aforementioned C-type models, integrating leakage loop and variable $P_{S R}$ computational algorithms, has been proposed by Alimhillaj and Dalla Vedova (2018) and named model C4. This model tried to integrate into the same transfer function the effects because of the interaction between the saturation block of the differential pressure and the leakage loop. Unfortunately, although involving 
an important increase in the complexity of the algorithm and computational burdens, the model $\mathrm{C} 4$ does not bring any performance improvement, providing results almost identical to the model C2 (Dalla Vedova et al., 2019).

Another formulation (named $\mathrm{C} 5_{\mathrm{OLD}}$ ), derived from the previous C-type models, is also proposed by Dalla Vedova et al. (2019). Model C5 $5_{\mathrm{OLD}}$ is intended to overcome the problems related to the interaction between the pressure saturation block and the leakage feedback loop by modifying the formulation of the pressure/flow gain ratio $G_{P Q}$ introduced in the $\mathrm{C} 3$ model. Unfortunately, this solution simulates more realistically the cases of high spool displacement but, it is still unsatisfactory in fine regulation (i.e. for small spool openings around its centered position). Therefore, the so-obtained EHSV fluid dynamic characteristic is acceptable for large spool displacements, i.e. $\left|x_{s}\right| \gg x_{s s}$, but also the C5 OLD model is not able to simulate the effects of the water hammer. However, these models do not lead to appreciable improvements (compared to the previously illustrated algorithms). Still, they raise the complexity of the algorithms and, especially in the C4 model, considerably increase the computational costs (computation time). Therefore, we have decided to leave out the $\mathrm{C} 4$ and $\mathrm{C} 5_{\mathrm{OLD}}$ models from this work.

\section{Proposed electrohydraulic servovalves numerical model}

The new EHSV numerical model proposed by authors in this work (i.e. model C5) was derived from a previous variant of the above C-type models (called model $\mathrm{C} 5_{\mathrm{OLD}}$ ) formerly proposed by Dalla Vedova et al. (2019). The leakage loop's location entirely downstream or upstream of the pressure saturation block, as implemented in the former C-type models, does not give a sufficiently close representation of the actual physical phenomena. As illustrated in Section 4, the mutual position of the saturation block and the feedback and leakage rings characterize the behavior of the different models considered (and their limits). However, placing the pressure saturation downstream of the flow

Figure 3 Conceptual block diagrams of the proposed EHSV model

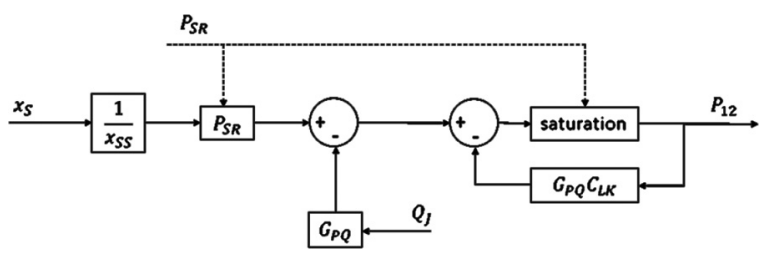

Figure 4 Intermediate formulations of the proposed EHSV model

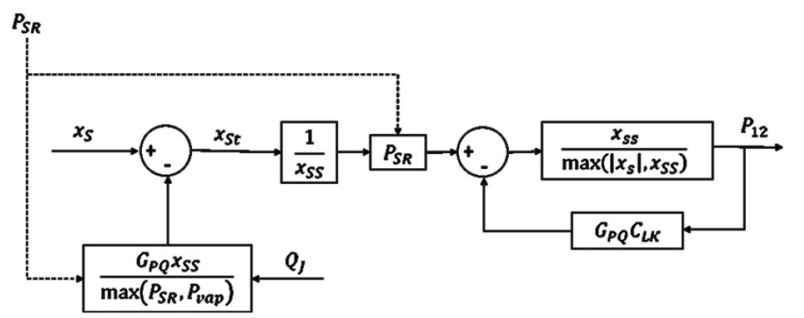

(a) feedback, none of the previous solutions can satisfactorily estimate the fine regulation conditions with non-zero flow rates and simulate water hammers. In model $\mathrm{C} 5$, the authors overcome these problems starting from the conceptual block diagram proposed by Alimhillaj and Dalla Vedova (2018) by pre-solving the leakage ring and the saturation block as if they were a nonlinear instantaneous feedback ring (as shown in Figure 3).

We can modify the block diagram shown in Figure 3 by shifting the gain $P_{S R} / x_{S S}$ downstream of the flow counterreaction node and implementing the $P_{12}$ differential pressure saturation block according to the model proposed by Dalla Vedova et al. (2019). In this way, we can obtain the block diagram shown in Figure 4(a).

Therefore, referring to the block diagram shown in Figure 4, we can state that the differential pressure $P_{12}$, regulated by the displacement of the valve spool, is also influenced by the flow of oil QJ passing through the valve ports (i.e. flow feedback) according to Borello and Villero (1991).

To this purpose, authors introduced an equivalent spool position $x_{S t}$, which can take into account the fluid flow through the valve and the potentially variable supply pressure as follows:

$$
x_{S t}=x_{S}-\frac{Q_{f} G_{P Q} x_{S S}}{\max \left(P_{S R}, P_{v a p}\right)}
$$

As can be seen from equation (3), the effect of the flow counterreaction (i.e. the feedback effect because of the flow of oil $Q_{f}$ crossing through the regulation ports of the EHSV) is gradually more relevant as the supply pressure decreases (up to the corresponding vapor tension value); in other words, it is equivalent to reduce the effect of the flow feedback with $P_{S R}$, down to the vapor pressure of the hydraulic fluid $P_{\text {vap }}$. Then, by pre-solving the instantaneous algebraic loop consisting of the leakage ring and the saturation block, we can replace it with the transfer function shown in Figure 4(b).

The proposed C5 model calculates the differential pressure $P_{12}$ as a function of the aforesaid equivalent spool position $x_{S t}$ and the variable pressure gain $P_{S R} /\left(\max \left(\left|x_{S}\right|, x_{S S}\right)+G_{P Q} C_{L K}\right.$ $x_{S S}$ ), sensitive to the combined effects of variable supply pressure $\left(P_{S R}\right), P_{12}$ saturation, and leakages:

$$
P_{12}=x_{S t} \frac{P_{S R}}{\max \left(\left|x_{S}\right|, x_{S S}\right)+G_{P Q} C_{L K} x_{S S}}
$$

As a result, the effect of the leakage flow is evaluated in a manner similar to model $\mathrm{C} 3$, by considering the reciprocal interaction between pressure saturation and leakages but resulting more consistently with the HF model (or experimental data). The soobtained model is shown in the block diagram of Figure 5.

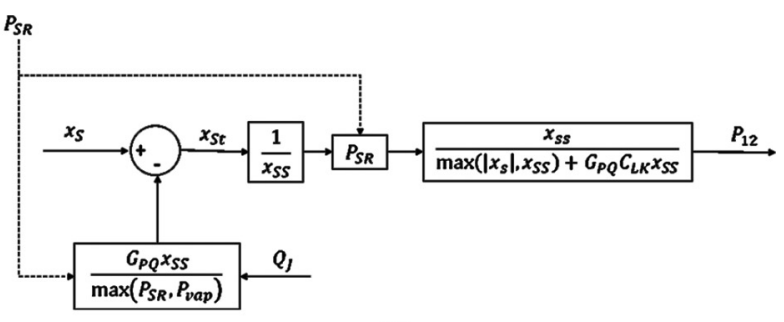

(b) 
Figure 5 New simplified numerical model C5 simulating the EHSV fluid dynamic behaviors

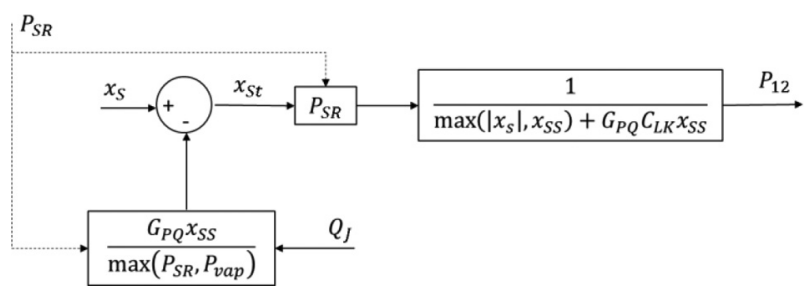

\section{Analysis of fluid dynamic characteristic of the considered models}

In this section, a preliminary evaluation of the C5 fluid dynamics model is proposed; it is performed on the basis of the corresponding EHSV fluid dynamic characteristics $P_{12}$ vs $\left(x_{S}, Q_{7}\right)$.

These diagrams show the differential pressure $P_{12}$ (also sensitive to supply pressure $P_{S R}$ and losses because of leakage) as a function of the valve spool displacement $x_{S}$, parameterized in the flow rate $Q_{f}$. The five fluid dynamic characteristics of the simplified models A, C1, C2, C3 and C5 have been computed referring to a EHSV featuring a value of $x_{S S}=0.1 \mathrm{~mm}, G_{P Q}=$ $7.4 \cdot 10^{11} \mathrm{~Pa} \cdot \mathrm{s} / \mathrm{m}^{3}$ and

$C_{L k}=2 \cdot 10^{-13} \mathrm{~m}^{3} / \mathrm{s} / \mathrm{Pa}$, in presence of a supply pressure $P_{S R}=20 \mathrm{MPa}$.

The so-obtained maps, respectively depicted in Figure 6(a)-6(e), can be compared to that of the HF model, shown in Figure 6(f), to evaluate the performances of the related EHSV fluid dynamic models. The comparison with the HF map puts in evidence how none of the preexisting simplified models can adequately simulate the real system (and, above all, the effect of water hammers). It should be noted that only the C5 model, albeit with some limitations because of the relatively simplified model, is able to approximate the main nonlinear behaviors simulated by the $\mathrm{HF}$ model e.g. spool fine regulation, water hammer, differential pressure saturation and correct estimation of leakages.

As shown in Figure 6(f), in the case of large spool displacement $\left(\left|x_{S}\right| \gg x_{S S}\right)$, the pressure drops because of the leakages, and the flow counterreaction are negligible. Therefore, the differential pressure $P_{12}$ regulated by the EHSV at its delivery ports tends asymptotically to the hydraulic system pressure PSR (consistently with the experimental evidence reported by Pace et al., 2013).

Consistently with the literature, model A (devoid of pressure saturation) differs significantly from $\mathrm{HF}$, highlighting all the limits of its linear formulation. In particular, being linear, model A cannot account for the $P_{12}$ pressure saturation at $\pm P_{S R}=20 \mathrm{MPa}$. Indeed, for $\left|x_{S}\right| \gg x_{S S}$, it calculates differential pressure values $P_{12}$ that, in absolute value, can be much higher than the actual supply pressure $P_{S R}$ provided by the hydraulic system (even in case of zero flow conditions).

To overcome this flaw and simulate the saturation of the differential pressure p12 that occurs with large spool openings, the former C-type models directly implement a saturation block downstream of the flow feedback, as shown in Figure 2(b), 2(c) and $2(\mathrm{~d})$.

However, the above differential pressure saturation conditions are satisfactorily estimated only by models C2, C3 and C5. Instead, in this case, the C1 model (generally) deviates from the other models, underestimating the pressure mentioned above. Indeed, as shown in Figure 2(b), in model $\mathrm{C} 1$, the leakage block is placed downstream of the pressure saturation. Therefore, model C1 can correctly calculate this $P_{12}$ only in the absence of leakages $\left(C_{L k}=0\right)$. Instead, in the presence of leakages (generally between spool and sleeve), it underestimates the said saturation pressure.

Regarding the operating conditions of the EHSV in fine adjustment with non-zero flow rates (and any water hammer), the $\mathrm{A}, \mathrm{C} 1$ and $\mathrm{C} 2$ models do not provide satisfactory results. In fact, by calculating the saturation of the differential pressure $P_{12}$ downstream of the feedback node in flow rate, any overpressure due to the oil flow rate that flows through the adjustment ports (with a small passage area because $\left|x_{S}\right|<x_{S S}$ ) is limited at the corresponding saturation pressure.

Even the C3 model, for small spool displacement, calculates a differential pressure trend that does not at all reflect that simulated by the HF. Despite the modified formulation to account for the pressure saturation within the leakage flow evaluation (as shown in subsection 4.4), the saturation block located upstream of the pre-solved leakage loop cuts any pressure peak higher than $P_{S R}$ [Figure 2(b)].

The shortcomings found in the former C-type models are partially overcome by the modification introduced with the model C5, as shown in Figure 6(e). Despite some differences attributable to the linearized approach adopted, the C5 model can approximate the HF model map with sufficient accuracy, simulating water hammers and the nonlinear dependency of the differential pressure from the spool displacement.

\section{Analysis of the numerical results provided by electrohydraulic actuator test bench}

The valve simplified models have been further tested by integrating them into a virtual test bench, which simulates the full position control electrohydraulic servoactuator shown if Section 3.

This EHA numerical model, proposed by Alimhillaj et al. (2013), considers the leading electrical, hydraulic and mechanical characteristics of the real system, including inertia, viscous and dry frictions acting on the hydraulic piston and a third-order electromechanical model of the EHSV. The simulations shown in Figure 7(a)-7(d) represents the dynamic response of the EHA to a combination of position command inputs (Com), external loads $\left(F_{R}\right)$ and variations in the hydraulic supply pressure $\left(P_{S R}\right)$, for the different models of the second stage sliding spool valve. The input sequence was defined to highlight the performance of the proposed fluid dynamics models and their effect on the behavior of the simulation test bench (Alimhillaj et al., 2013). The response of the EHA equipped with the HF model is shown in Figure 8, for comparison with the simplified models.

The Com timeline is composed by a sequence of three stepping inputs, respectively, from $0 \mathrm{~m}$ (initial position) to $0.02 \mathrm{~m}$ at time $=0 \mathrm{~s}$, from $0.02 \mathrm{~m}$ to 0.03 at time $=0.3 \mathrm{~s}$ and finally from $0.03 \mathrm{~m}$ to $0.02 \mathrm{~m}$ at time $=0.75 \mathrm{~s}$. The external load $F_{R}$ is null from $0 \mathrm{~s}$ to $0.2 \mathrm{~s}$. Subsequently, it instantaneously reaches its final value $(10400 \mathrm{~N})$. In this way, the first EHA run is an unloaded actuation. On the contrary, during the second (starting at time $=0.3 \mathrm{~s}$ ) and the third 
Figure 6 Fluid dynamic characteristic $P_{12}=f\left(X_{S}, Q_{J}\right)$ of the considered EHSV simplified models

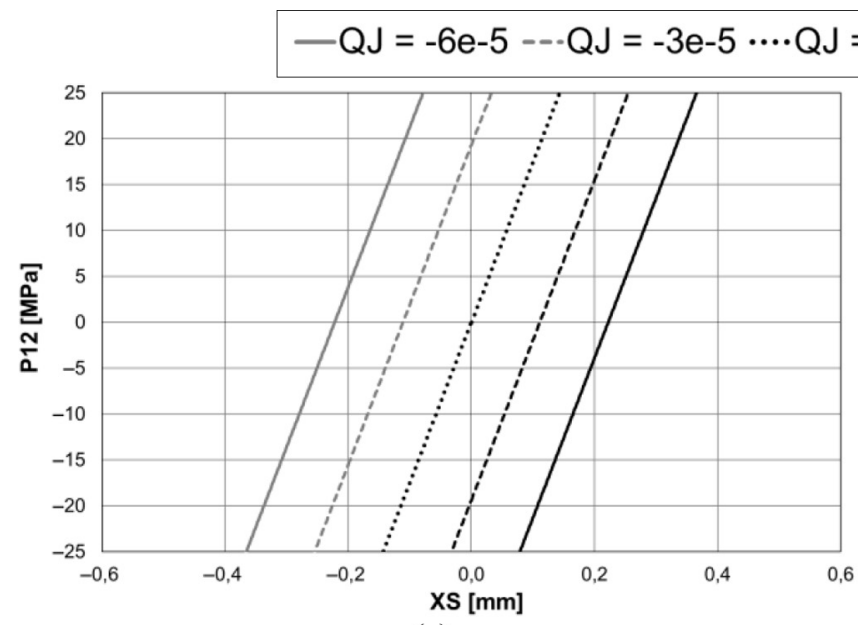

(a)

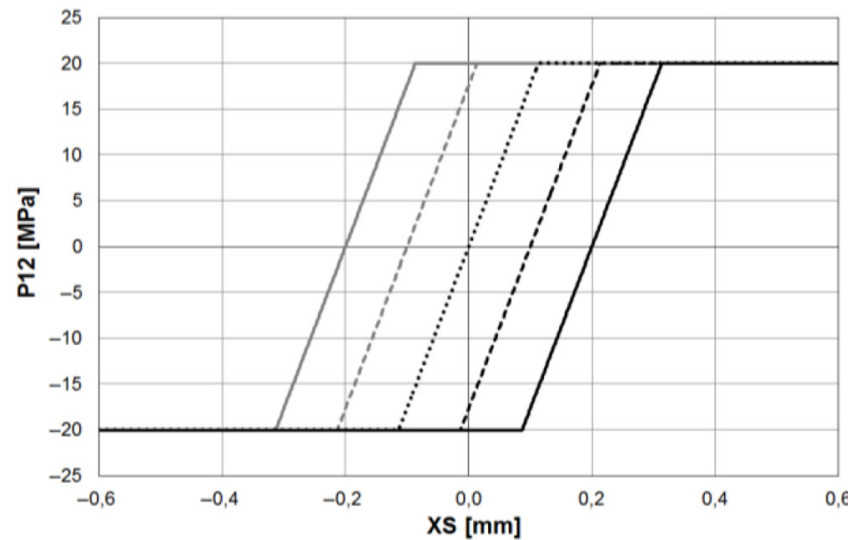

(c)

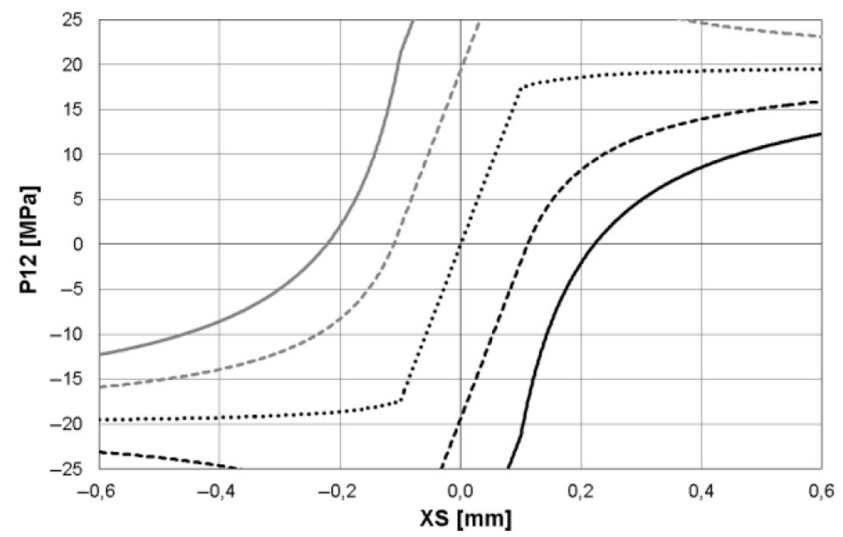

(e)

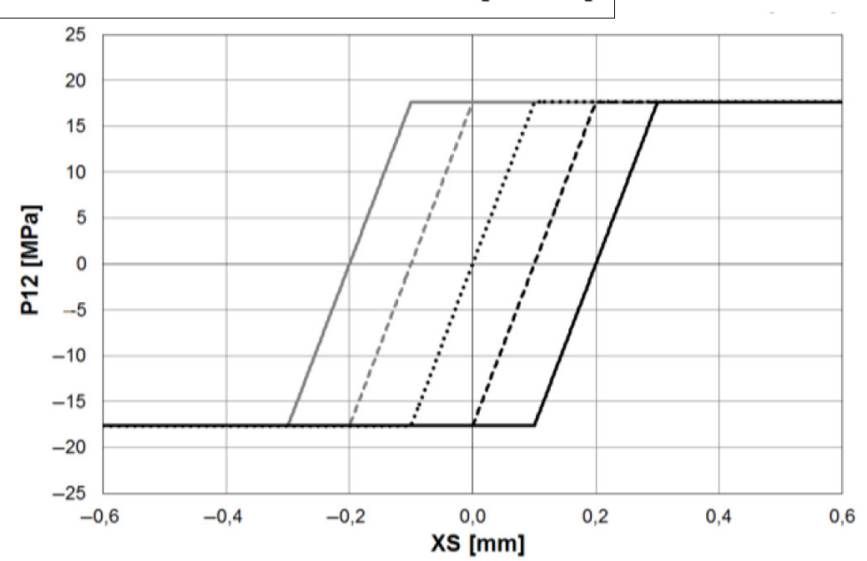

(b)

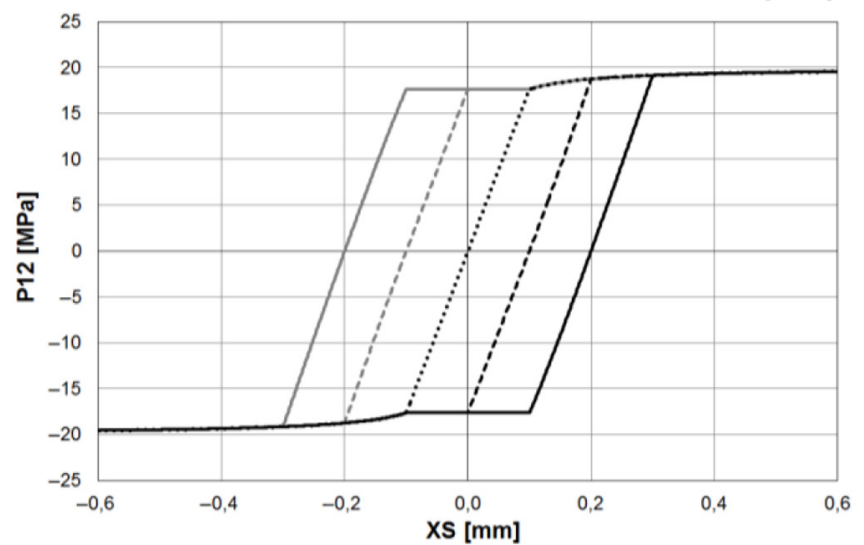

(d)

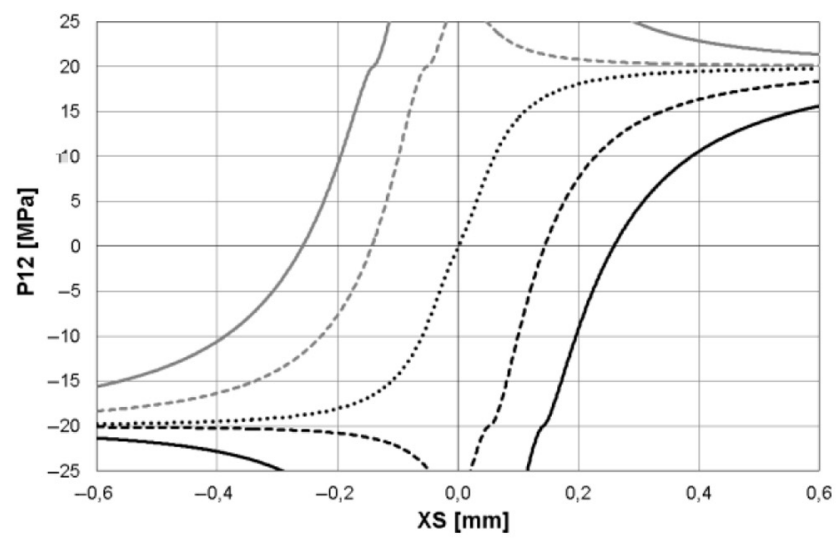

(f)

Notes: Pressure-flow-spool position maps $-C_{L K}=2 \cdot 10-13\left[\mathrm{~m} 3 / \mathrm{s} / P_{a]}-\mathrm{PSR}=20[\mathrm{MPa}]-x_{S S}=0.1[\mathrm{~mm}]\right.$. (a) model A; (b) model Cl; (c) model C2; (d) model C3; (e) model C5; and (f) model HF

actuation runs (time $=0.75 \mathrm{~s}$ ), $F_{R}$ acts, respectively, as opposing or aiding load. The $P_{S R}$ timeline is composed of three time intervals, each characterized by a constant differential pressure value. During the first and the third time interval (respectively, from $0 \mathrm{~s}$ to $0.35 \mathrm{~s}$ and from $0.45 \mathrm{~s}$ to the end of simulation), the supply pressure keeps its nominal value of $20 \mathrm{MPa}$ (corresponding stall load $F_{R}=14.1 \mathrm{kN}$ ). Instead, during the second time interval $(0.35 \mathrm{~s}$ to $0.45 \mathrm{~s})$, it decreases instantaneously to $12 \mathrm{MPa}$ (related stall load $F_{R}=8.5 \mathrm{kN}$ ). In this way, we also evaluated the effect of a temporary supply 
Figure 7 EHA test bench simulation with the considered EHSV simplified models

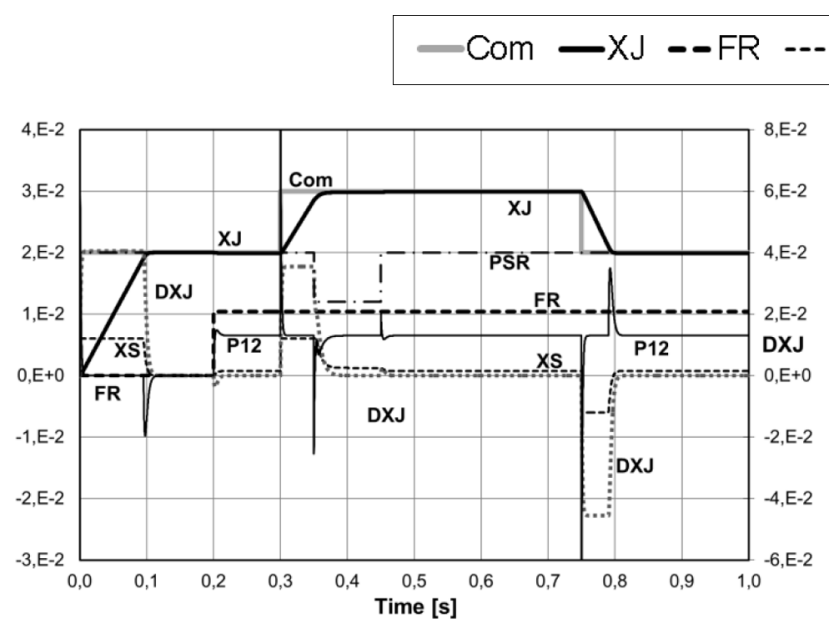

(a)

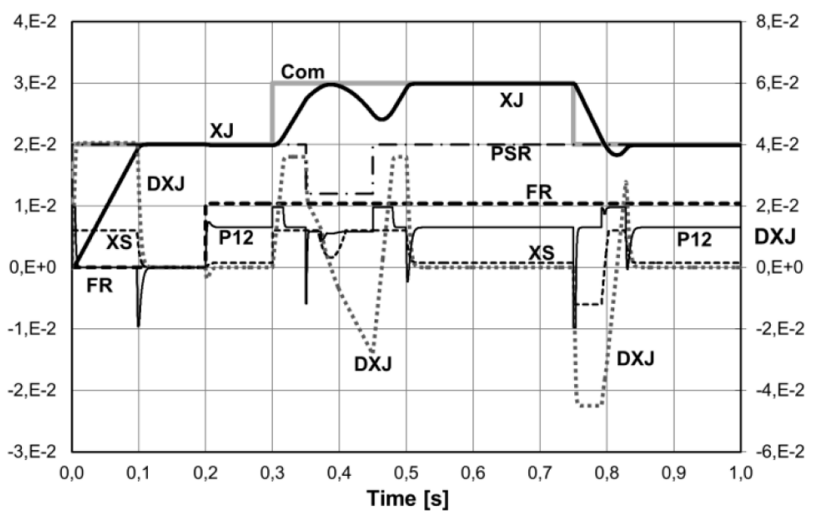

(c)

Notes: (a) Model A; (b) model C1; (c) model C3; (d) model C5

Figure 8 EHA test bench simulation with the EHSV HF model

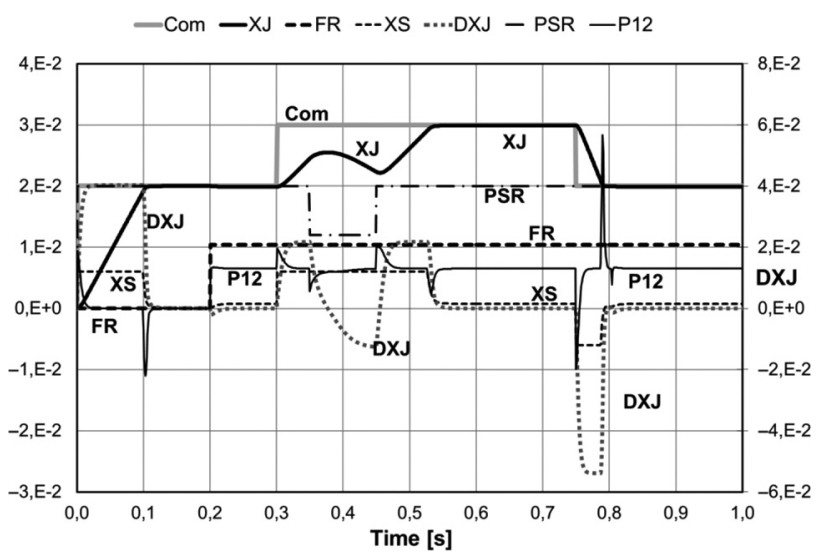

pressure drop (i.e. during the actuation run of the EHA jack under opposing load).

All the considered C-type models provide satisfying simulations of the unloaded actuation run. Similar thoughts apply for the actuation run with aiding load (i.e. the sections of

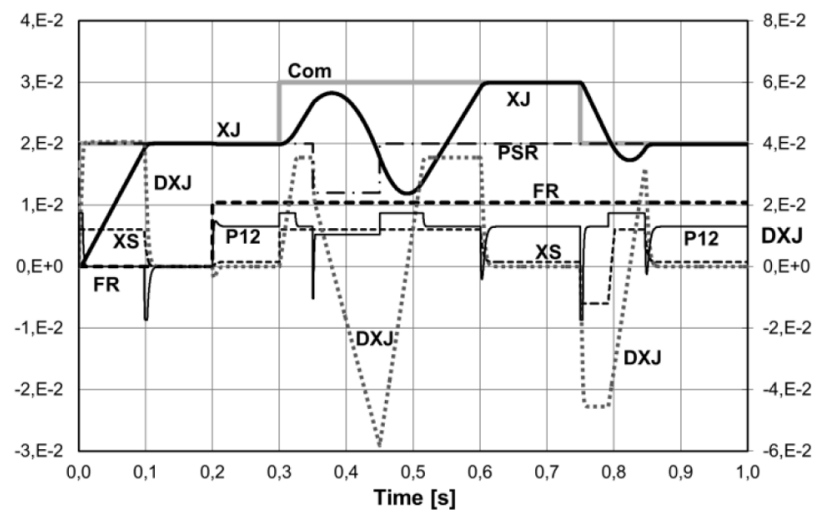

(b)

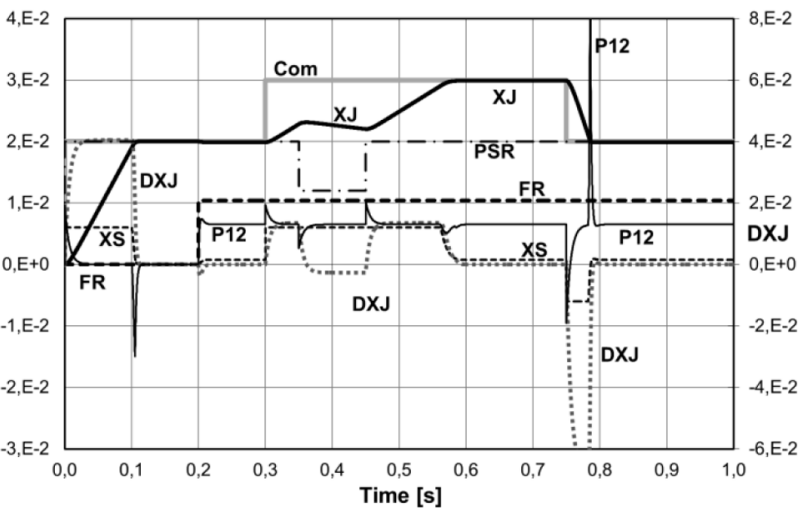

(d)

the curves $X_{\mathcal{f}}$ starting at time $=0.75 \mathrm{~s}$ ). On the contrary, in the case of opposing load, the simulated actuation runs represented in Figure 7(a)-7(d) shows several incongruences with the HF reference of Figure 8, putting in evidence some shortcomings previously highlighted for models A, C1 and C5.

Regarding model A [Figure 7(a)], we can see how the opposing load effect on the actuation rate is underestimated. Indeed, when the supply pressure drops, the system's back movement is absent (unlike the HF case). It is due to the inability of model A to compute the $P_{12}$ saturation conditions.

Regarding model C1 [Figure 7(b)], the actuation run with opposing load shows a significant incongruence with the $\mathrm{HF}$ reference. In terms of speed reduction, the load effect on the system actuation velocity is underestimated (as in model A). Having the leakage loop downstream of the saturation block ([Figure 2(b)], this model underestimates the saturation differential pressure value. In conditions of actuation with opposing load and supply pressure drop, this determines the development of an actuation force lower than $F_{R}$. Therefore, when the supply pressure drops, the system's backward movement is overrated, simulating a wrong constant backward acceleration.

Model C3 [Figure 7(c)] simulates with sufficient accuracy the behavior of the HF model for the unloaded actuation (from 
0 to $0.2 \mathrm{~s}$ ) and the aiding load condition (at $0.75 \mathrm{~s}$ ), despite a slightly lower stopping deceleration (because of the inability of model C3 to simulate the water hammer effect). This shortcoming, which emerges from the comparison with the $\mathrm{HF}$ model, is the cause of the overshoot phenomena during the actuation stroke following the second command step (time $\geq 0.3 \mathrm{~s}$ ) and in the subsequent actuation with aiding load (time $\geq 0.75 \mathrm{~s}$ ). This effect is more evident in the second actuation run (time $\geq 0.3 \mathrm{~s}$ ), in which the temporary drop in the $P_{S R}$ supply pressure makes the deceleration of the actuator even less sudden (causing the large $x_{\mathcal{f}}$ overshoot at time $=0.4 \mathrm{~s}$ ).

In general, model C5 [Figure $7(\mathrm{~d})$ ] reproduces all the working conditions simulated by the considered EHA numerical test bench with a suitable fidelity to the corresponding HF reference. However, also in this case, we found some unavoidable deficiencies connected to the model C5 simplified formulation. Indeed, despite having a more complex nonlinear formulation than the previous type $\mathrm{C}$ models, C5 simulates the EHSV fluid dynamic behaviors using only a few parameters easily detectable experimentally (e.g. $G_{P}$, $G_{Q}, C_{L k}$ and $\left.x_{S S}\right)$. On the contrary, to simulate the fluid dynamics behavior of the EHSV, the HF model needs a large amount of physical and geometrical parameters such as density and viscosity of the hydraulic fluid, supply-side overlap, returnside overlap, radial gap, circumferential passage width, throttle area shape, control lands radius and eventual spool eccentricity. Furthermore, despite having a nonlinear formulation, model C5 implements a linear relationship between flow and pressure losses across the valve passageways. It is very compact and requires a low computational effort (few elementary instructions, pre-solved instant models, no algebraic loops or other numerical troubles). Instead, the $\mathrm{HF}$ reference implements a more realistic linear-quadratic fluid dynamic model, particularly useful in the medium-high flow rates but gives higher computational costs (as shown by Borello et al., 2009). Therefore, as can be seen by comparing Figures 7 (d) and 8 , model $\mathrm{C} 5$ simulates the unloaded actuation rate correctly but underestimates it in case of opposing load and overestimates it in case of aiding load; in conditions of opposing load and supply pressure drop, when the external load $F_{R}$ overcomes the actual value of the piston stall load (depending on P12) developing an over-stall condition, the back velocity computed in steady condition by model C5 is lower than given by HF. This difference is due to using a linear damping law (model C5) instead of a quadratic (HF model in case of turbulent regime). The linear relationship between pressure drop and flow rate, in fact, overestimates the damping action in medium-low flow conditions and underestimates it in mediumhigh regimes.

The merits of the considered fluid dynamics models can be quantified by assessing their consistency with the HF model. For this purpose, Figure 9 shows the normalized root mean square (RMS) error (NRMSE \%) values calculated by comparing the $\mathrm{HF}$ model time responses with those of the simplified models.

\section{Conclusions}

A new simplified model simulating the fluid dynamic behavior of a hydraulic servovalve is proposed. Its performance is evaluated by comparing other valve fluid dynamic numerical models (an $\mathrm{HF}$ and four simplified algorithms found in literature). The merits or demerits of all the above considered models, characterized by a semi-empirical formulation, are related to their ability to properly describe the fluid dynamic behavior of the valve, represented by the diagrams reporting their "characteristics" and by the simulations of a typical onboard servomechanism using it.

The results reported in this work show that none of those simplified models can accurately simulate the HF valve's behavior in its full operating envelope. Indeed, the HF model implements a nonlinear algorithm sensitive to the valve's internal geometry, fluid motion conditions (laminar or turbulent regime and related transitions) and oil characteristics (dynamic viscosity, temperature and contamination level). On the other hand, the C-type models describe the valve performance through different nonlinear formulations, all deriving from the same simplified model A (described in subsection 4.1). Their accuracy (ability to faithfully reproduce the HF system performance) is linked to the level of simplification and may vary depending on the operating conditions considered.

As regards the pressure-flow-spool position maps shown in Section 6, the first four fluid dynamics models (A, C1, C2 and C3) highlight their gaps by significantly deviating from the $\mathrm{HF}$ model. None of them can satisfactorily evaluate the fine regulation conditions (small spool displacements) with nonzero flows or simulate the water hammer phenomenon.

It must be noted that, although this condition rarely occurs in a hydraulic system's normal operation, a water hammer can significantly impact the actuator's behavior in the event of a pressure drop in the hydraulic supply. Several shortcomings are overcome by the proposed numerical model C5 that, albeit with some substantial differences attributable to the simplified approach adopted, can simulate some peculiar valve behaviors such as water hammer and nonlinear dependence of the

Figure 9 Normalized RMS error (NRMSE \%)
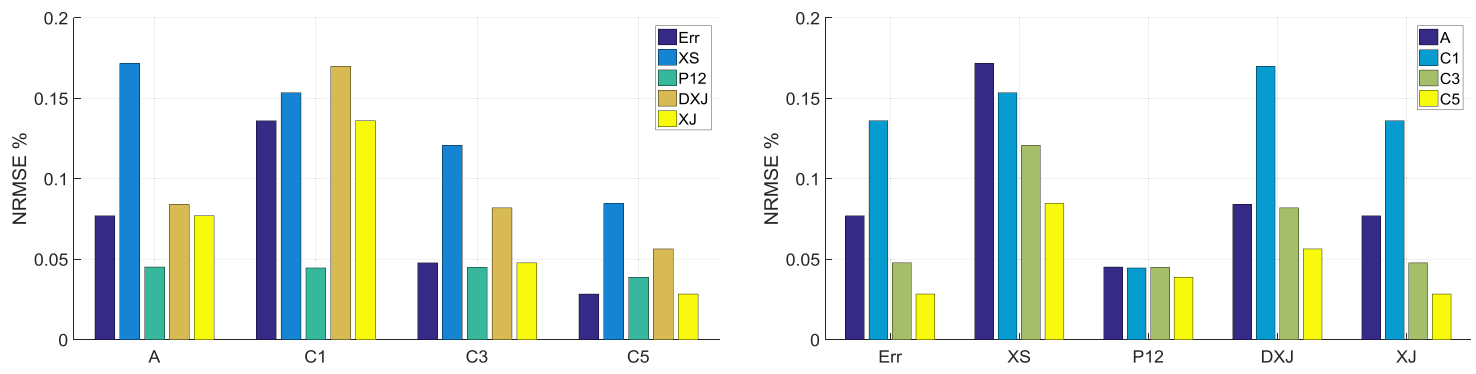
pressure with spool displacement. Therefore, on the basis of the results presented for the different models, it is advisable to evaluate in the future new formulations capable of simulating the fluid dynamic behavior of the valve with greater accuracy. However, some of the simplified models considered in this work can be effectively used in different activities (preliminary design, monitoring and prognostics). In particular, in such applications, the C5 model represents a useful trade-off between the algorithm's simplicity (few easily measurable parameters and reduced calculation times) and the accuracy of the results provided (compared to the HF model).

\section{References}

Alimhillaj, P. and Dalla Vedova, M.D.L. (2018), "Study of new fluid dynamic nonlinear servovalve numerical models for aerospace applications", Proceedings of the 2nd European Conference on Electrical Engineering and Computer Science (EECS), No. 8910132, pp. 483-490.

Alimhillaj, P., Borello, L. and Dalla Vedova, M.D.L. (2013), "Proposal of innovative fluid dynamic nonlinear servovalve synthetic models", International fournal of Mechanics and Control, Vol. 14 No. 2, pp. 39-49.

Berri, P.C. and Dalla Vedova, M.D.L. (2020), "A review of simplified servovalve models for digital twins of electrohydraulic actuators", Fournal of Physics: Conference Series, Vol. 1603 No. 12016.

Borello, L. and Villero, G. (1991), "Comparison of simplified models of control valves (confronto fra modelli semplificati di valvole di commando)", Proceedings of the XI A.I.D.A.A. Congress.

Borello, L., Dalla Vedova, M.D.L., Jacazio, G. and Sorli, M. (2009), "A prognostic model for electrohydraulic servovalves", Proceedings of the Annual Conference of the Prognostics and Health Management Society (PHM 2009).

Byington, C.S., Watson, M., Edwards, D. and Stoelting, P. (2004), "A model-based approach to prognostics and health management for flight control actuators", 2004 IEEE Aerospace Conference Proceedings, Vol. 6, pp. 3551-3562.

Chen, Q. and Stoffel, B. (2004), "CFD simulation of a hydraulic servo valve with turbulent flow and cavitation", ASME/FSME 2004 Pressure Vessels and Piping Conference.

Dalla Vedova, M.D.L., (2007), "Ideation of advanced physical-mathematical models for the simulation and design of flight controls and related study of innovative architectures", PhD thesis, Politecnico di Torino, Turin.

Dalla Vedova, M.D.L., Maggiore, P. and Pace, L. (2014), "Proposal of prognostic parametric method applied to an electrohydraulic servomechanism affected by multiple failures", WSEAS Transactions on Environment and Development, Vol. 10, pp. 478-490.

Dalla Vedova, M.D.L., Berri, P.C., Corsi, C. and Alimhillaj, P. (2019), "New synthetic fluid dynamic model for aerospace four-ways servovalve", International fournal of Mechanics and Control, Vol. 20 No. 2, pp. 105-112.
De Martin, A., Dellacasa, A., Jacazio, G. and Sorli, M. (2018b), "High-Fidelity model of electro-hydraulic actuators for primary flight control systems", Proceedings BATH/ASME Symp. Fluid Power and Motion Control.

De Martin, A., Chiavaroli, P., Evangelista, G., Jacazio, G. and Sorli, M. (2018a), "Real time loading test rig for flight control actuators under PHM experimentation", Proceedings of the ASME 2018 International Mechanical Engineering Congress and Exposition.

Henninger, D., Zopey, A., Ihde, T. and Mehring, C. (2017), "High-fidelity 1D dynamic model of a hydraulic servo valve using 3D computational fluid dynamics and electromagnetic finite element analysis", International fournal of Mechanical and Mechatronics Engineering, Vol. 11 No. 8, pp. 1476-1482.

Jacazio, G. and Borello, L. (1986), “A non-linear model of an electro-hydraulic servo system with axial piston hydraulic motor", Proceedings 7th International Fluid Power Symposium, Bath.

Maré, J.C. (2016), Aerospace Actuators 1: Needs, Reliability and Hydraulic Power Solutions, ISTE and Wiley press, Vol. 1.

Maré, J.C. (2018), Aerospace Actuators 3: European Commercial Aircraft and Tiltrotor Aircraft, ISTE and Wiley press, Vol. 3.

Mehring, C., Zopey, A., Latham, M., Ihde, T. and Massie, M. (2014), "Decoupled 1D/3D analysis of a hydraulic valve", Int. Conf. of Comp. Methods in Sci. and Eng. 2014, AIP Conf. Proceedings, Vol. 1618, pp. 979-982.

Pace, L., Ferro, M., Fraternale, F., Caimano, A., Maggiore, P. and Dalla Vedova, M.D.L. (2013), "Comparative analysis of a hydraulic servo-valve", International Fournal of Fluid Power, Vol. 14 No. 2, pp. 53-62.

Urata, E. (2000), "Study of magnetic circuits for servovalve torque motors", Bath Workshop on Power Transmission and Motion Control, pp. 269-282.

Urata, E. (2001), "Study on leakage flux from servovalve torque motors", Proceedings Seventh Scandinavian Int. Conf. on Fluid Power, Vol. 1, pp. 51-66.

Urata, E. (2002), "Influence of fringing on servovalve torquemotor characteristic", Proceedings Fifth FFPS Intl. Symp. on Fluid Power, Vol. 3, pp. 769-774.

Urata, E. (2003), "Static stability of toque motors", The Eighth Scandinavian Intl. Conf. on Fluid Power, pp. 871-885.

Urata, E. (2004a), "Influence of eddy current on torque-motor dynamics", Proceedings 4th IFK Workshop, pp. 71-82.

Urata, E. (2004b), "One degree of freedom model for torque motor dynamics", International fournal of Fluid Power, Vol. 5 No. 2, pp. 35-42.

Urata, E. (2005), "Influence of asymmetry of air-gap in servovalve torque motor", 9th Scandinavian Int. Conf. on Fluid Power.

Yang, H., Wang, W. and Lu, K. (2019), "Cavitation and flow forces in the flapper-nozzle stage of a hydraulic servo-valve manipulated by continuous minijets", Advances in Mechanical Engineering, Vol. 11 No. 5, pp. 1-9.

\section{Corresponding author}

Matteo Davide Lorenzo Dalla Vedova can be contacted at: matteo.dallavedova@polito.it 